\title{
Upregulation of Mechanosensitive Channel Piezo1 Involved in High Shear Stress-induced Pulmonary Hypertension
}

\author{
Jiyuan Chen ${ }^{1}$, Jinrui Miao ${ }^{1}$, Jing Liao ${ }^{2}$, Dansha Zhou ${ }^{3}$, Ziyi Wang ${ }^{1}$, Ziying Lin ${ }^{4}$, Yuqin \\ Chen $^{5}$, Chenting Zhang ${ }^{4}$, Xiaoyun $\mathrm{Luo}^{6}$, Yi Li ${ }^{6}$, Yue Xing ${ }^{4}$, Manjia Zhao ${ }^{7}$, Sophia \\ Parmisano $^{7}$, Haiyang Tang ${ }^{1}$, Jason X.-J. Yuan ${ }^{8}$, Kai Yang ${ }^{5}$, Dejun Sun ${ }^{9}$, and Jian Wang ${ }^{7}$ \\ ${ }^{1}$ State Key Laboratory of Respiratory Disease \\ ${ }^{2}$ State Key Laboratory of Respiratory Disease, National Clinical Research Center for \\ Respiratory Disease, Guangdong Key Laboratory of Vascular Disease, Guangzhou Institute \\ of Respiratory Health, The First Affiliated Hospital of Guangzhou Medical University \\ ${ }^{3}$ First Affiliated Hospital of Guangzhou Medical University \\ ${ }^{4}$ First Affiliated Hospital of Guangzhou Medical College \\ ${ }^{5}$ Guangzhou Medical University \\ ${ }^{6}$ State Key Laboratory of Respiratory Disease, Guangzhou Institute of Respiratory \\ Disease, The First Affiliated Hospital of Guangzhou Medical University \\ ${ }^{7}$ University of California San Diego \\ ${ }^{8}$ Medicine Section of Physiology Division of Pulmonary, Critical Care and Sleep Medicine \\ Department of Medicine \\ ${ }^{9}$ The People's Hospital of Inner Mongolia
}

February 2, 2022

\begin{abstract}
Background and Purpose: Piezo1 is a crucial mechanical sensitive channel involved in vascular remodeling. However, the role of Piezo1 in different types of vascular cells during the development of pulmonary hypertension (PH) induced by high flow is largely unknown. Experimental Approach: Based on previously established protocols, we established a rat PH model by left pulmonary artery ligation (LPAL) for 2 and 5 weeks to mimic the high flow and hemodynamic stress. Key Results: Results showed that right ventricular systolic pressure (RVSP) and right ventricular wall thickness were significantly increased in the LPAL groups compared with the SHAM group. Rats in LPAL-5w groups developed remarkable pulmonary vascular remodeling, resulting in decreased phenylephrine-induced contraction and acetylcholine-induced relaxation. On the one hand, in pulmonary arterial smooth muscle cells (PASMCs), upregulation of Piezo1 was observed in association with the elevation of [Ca2+]cyt in the PASMCs from both LPAL-2w and LPAL-5w groups versus respective SHAM groups. Notably, Piezo1 expression was directly upregulated by YAP/TEAD4. On the other hand, significantly upregulated Piezo1 expression was also presented in the lung tissues, mostly composed of pulmonary endothelial cells (ECs), from rats of LPAL-2w and -5ws groups, which can be transcriptionally regulated by RELA (p65) and contributes to the lung inflammation. Conclusion and Implications: Our results suggested the upregulation of Piezo1 in both PASMCs and ECs, coordinate together and contribute to the pulmonary vascular remodeling and dysfunction in LPAL-PH rats, providing novel insights into the cell type-specific effects of Piezo1 in the pulmonary vasculature during high flow-related $\mathrm{PH}$.
\end{abstract}

\section{Hosted file}

manuscript-LPAL paper.doc available at https://authorea.com/users/458545/articles/555079- 
upregulation-of-mechanosensitive-channel-piezo1-involved-in-high-shear-stress-inducedpulmonary-hypertension
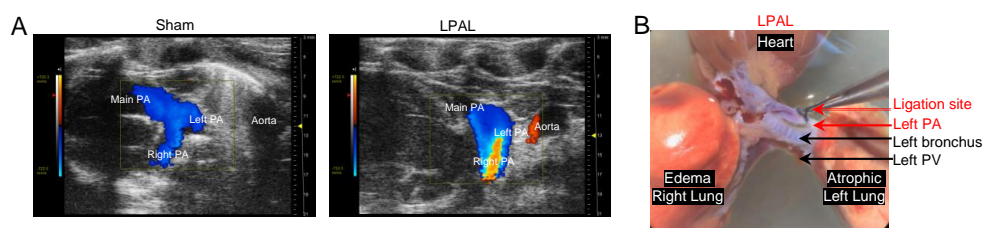

$\mathrm{C}_{\mathrm{a}}$

b
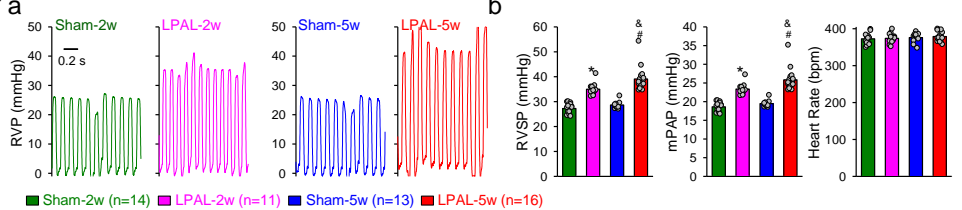

$\mathrm{D}$
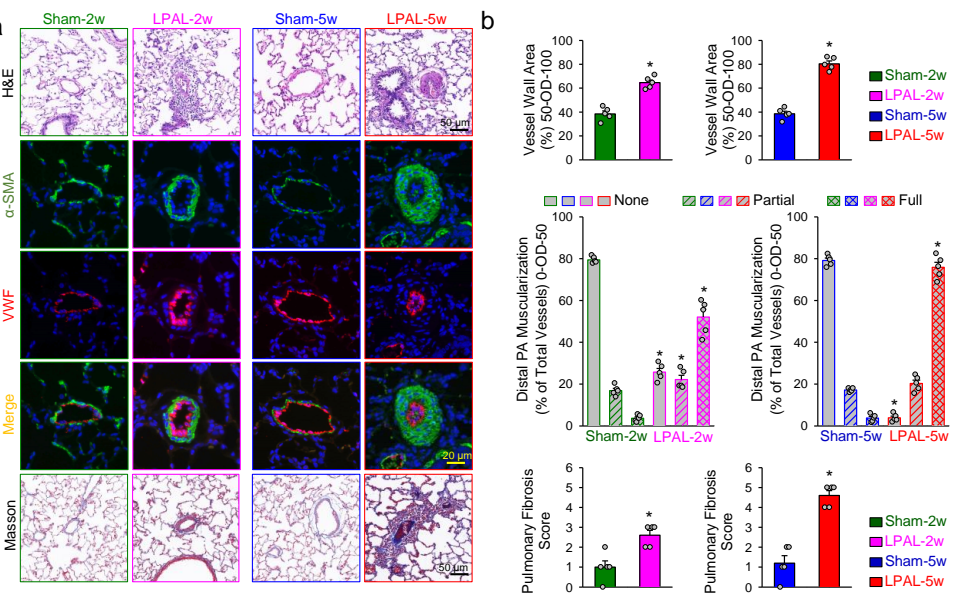

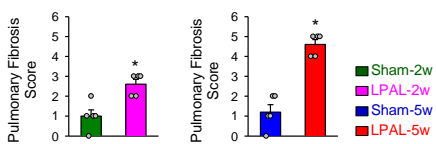

Figure 1 


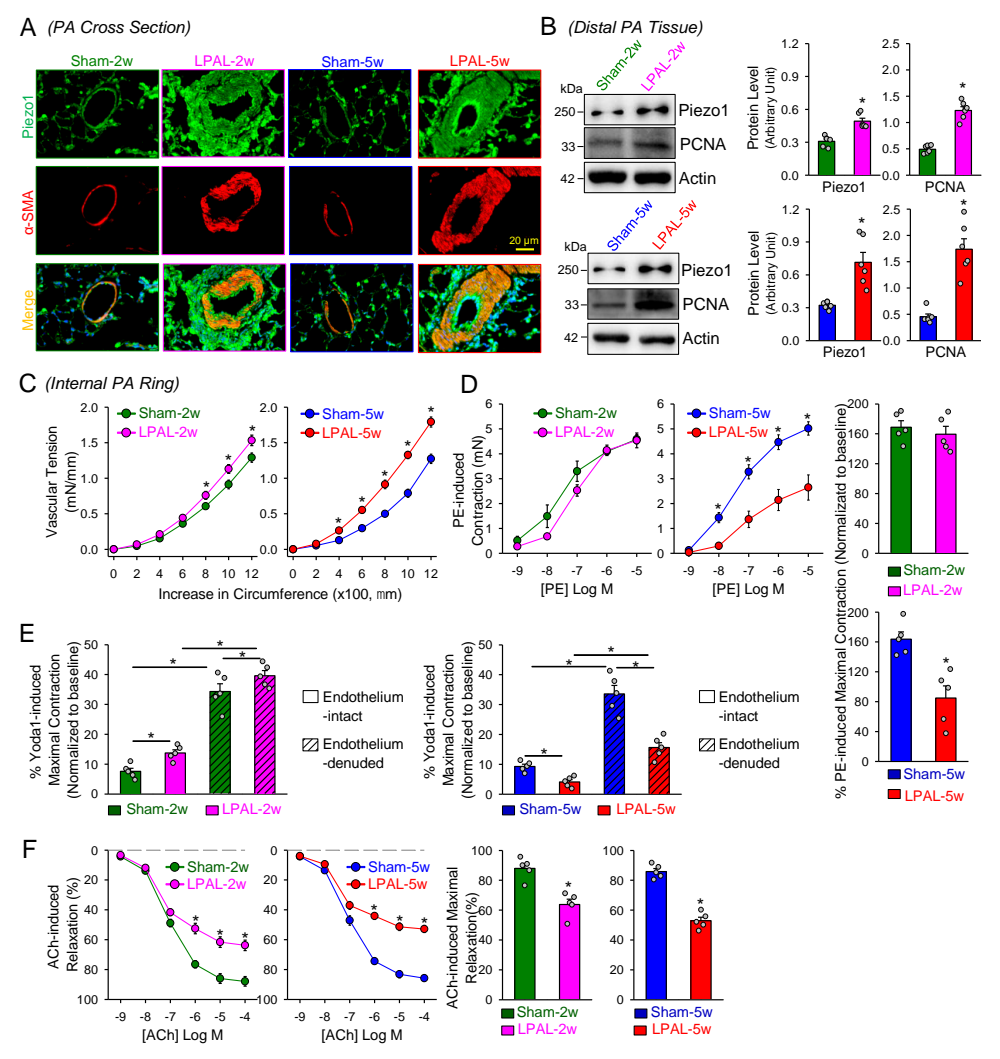

Figure 2 


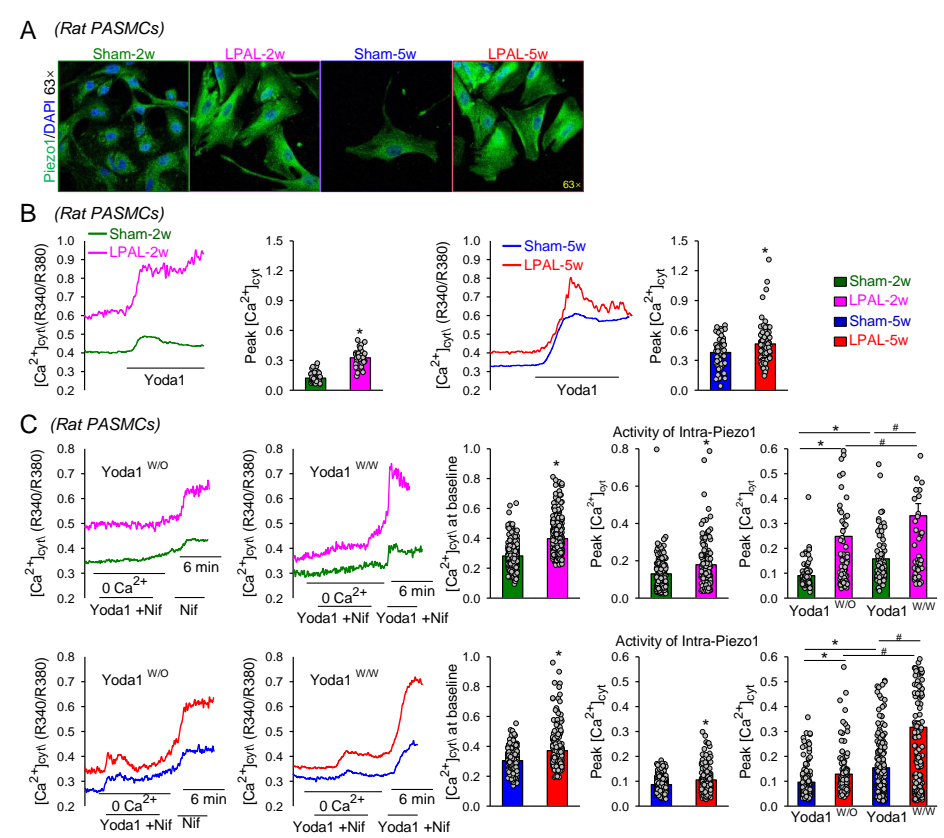

Figure 3 

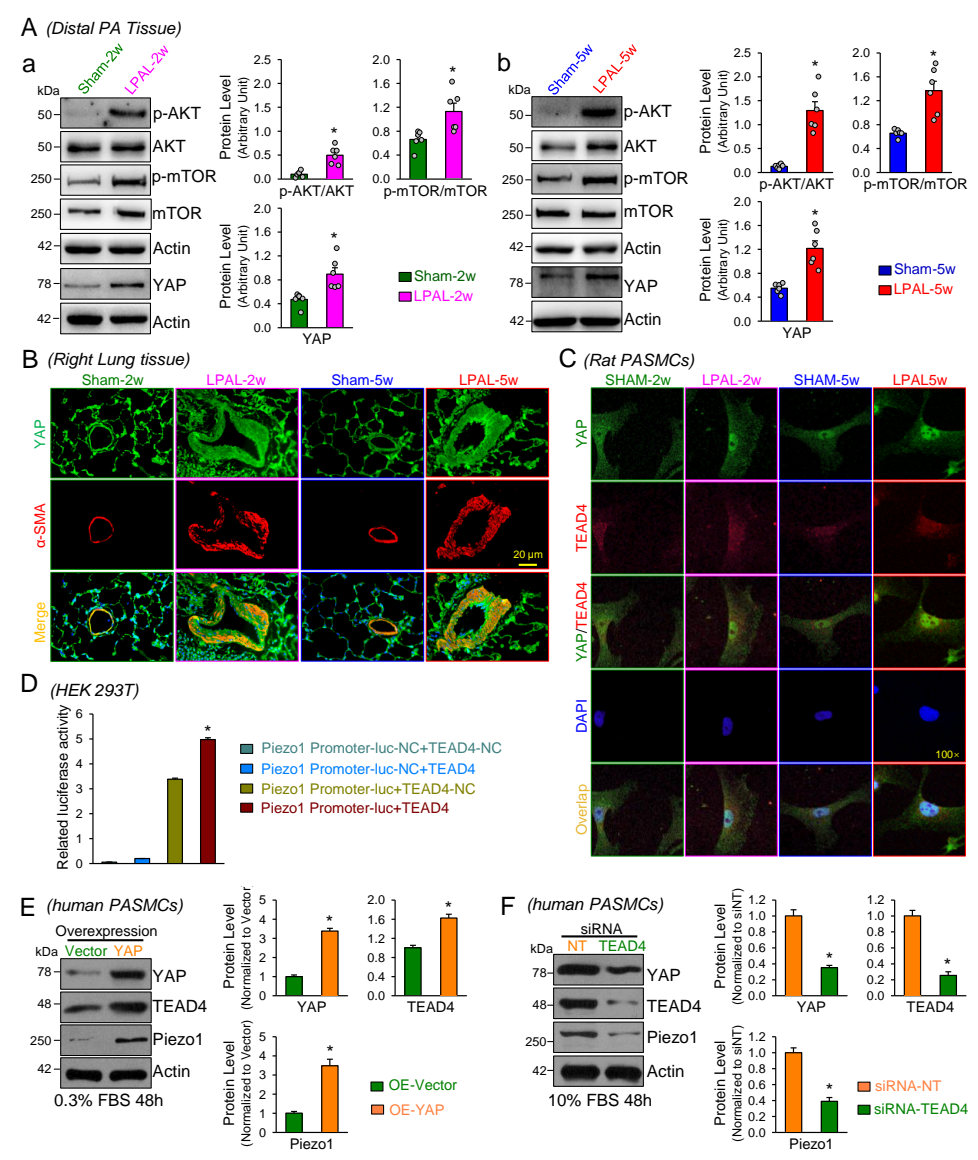

Figure 4 

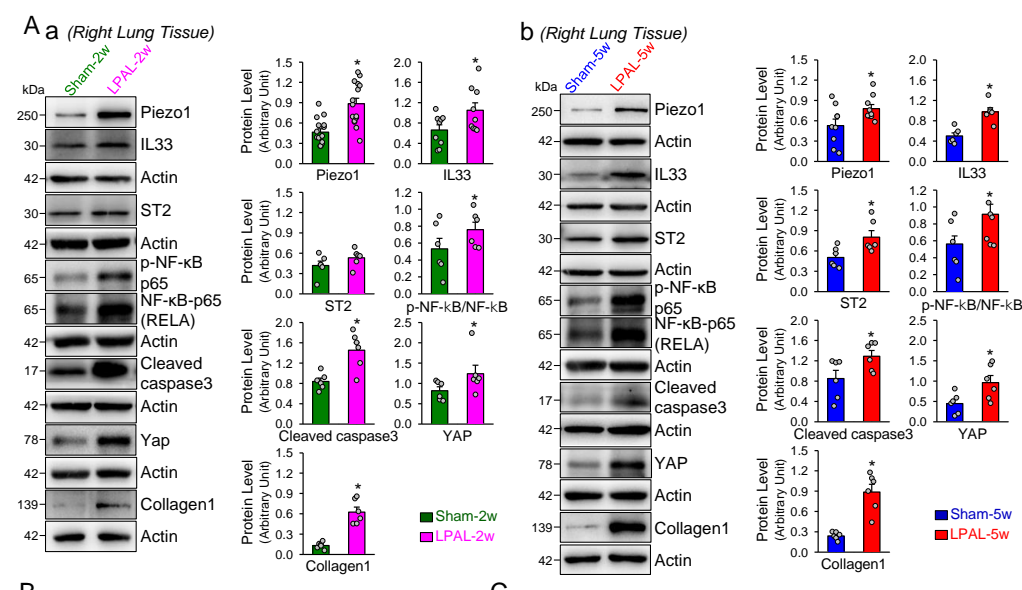

B (Right Lung Tissue)
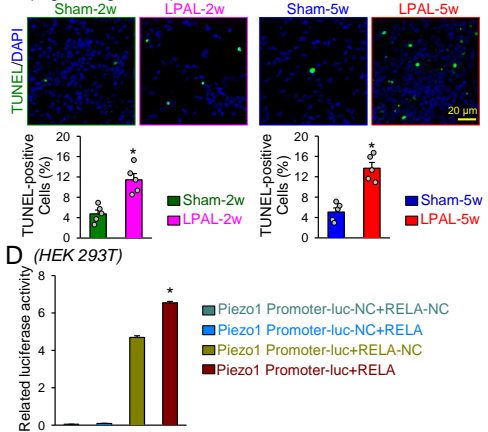

C (Right Lung Tissue)
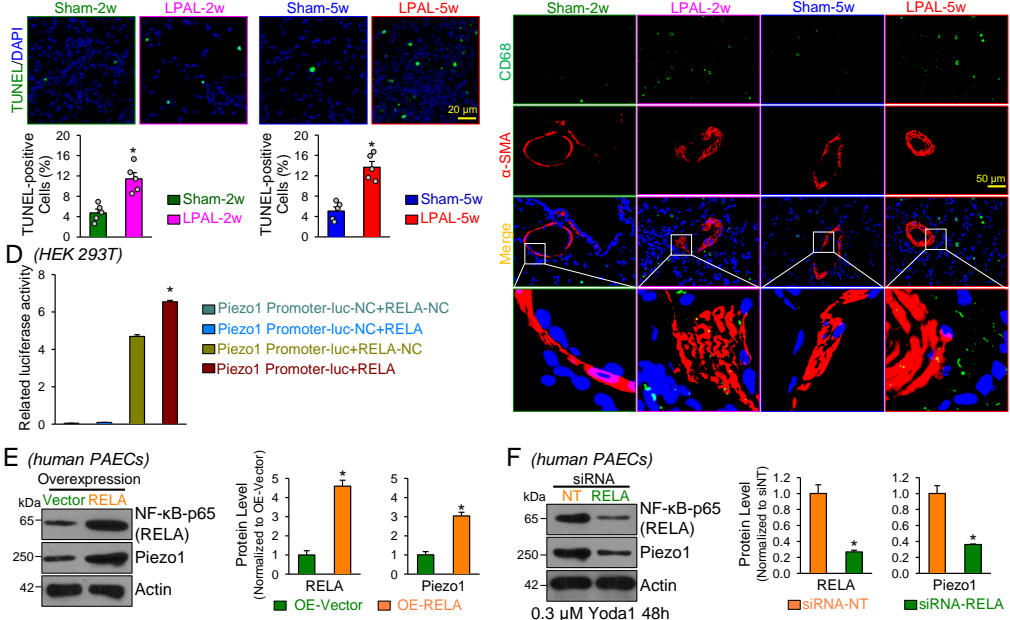

F (human PAECs)

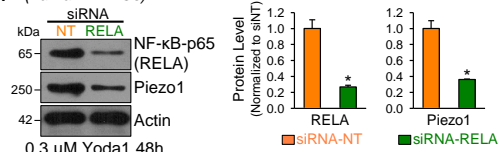

Figure 5 


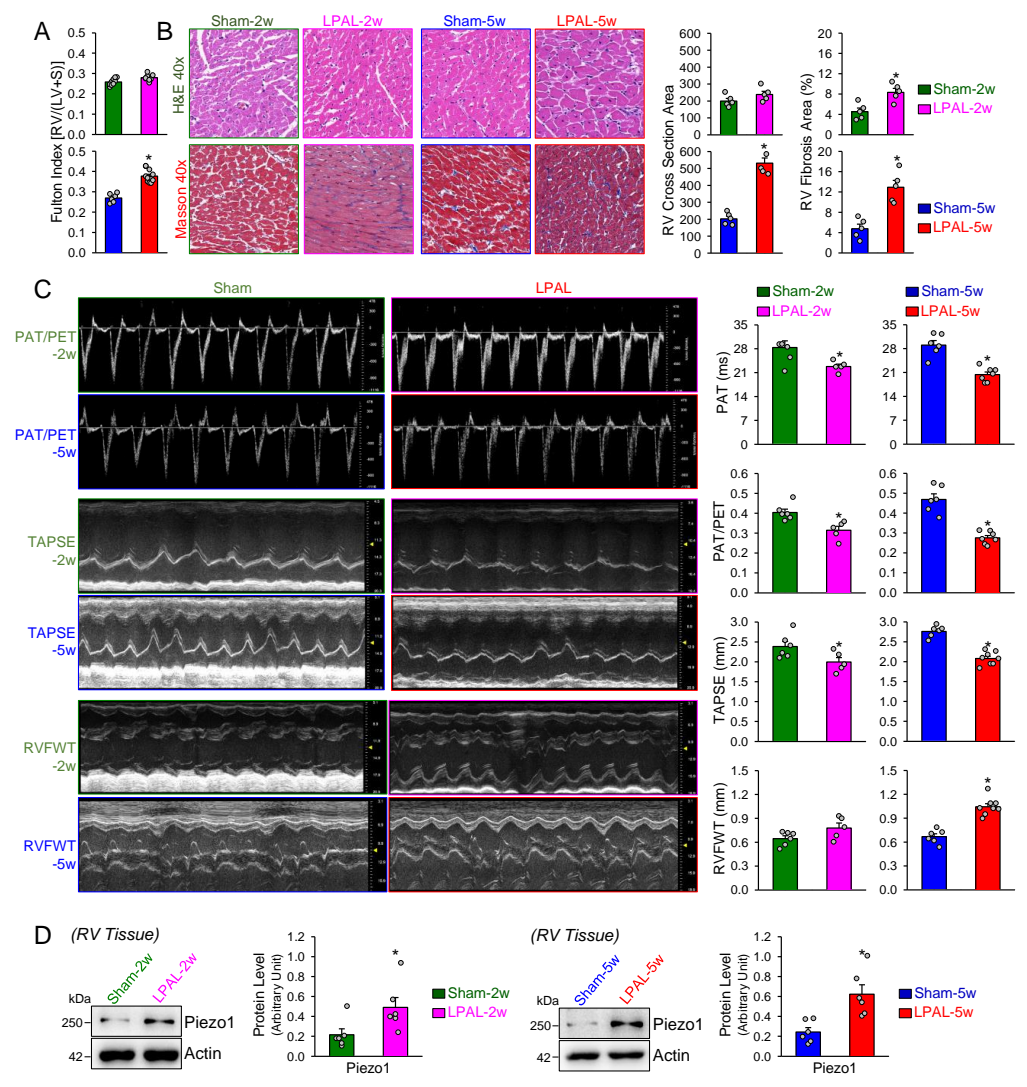

Figure 6 